\title{
The outcome of Flexor Tendon Repair based on the number of core suture, mechanism and zone of injury in Erbil
}

\section{Abstract}

Background and objective: Principles of surgical treatment of flexor tendon injuries include early primary repair with a strong core stitch combined with an epitendinous suture. This study aimed to evaluate the outcomes of primary flexor tendon repair according to the mechanism, zone of injury and number of core sutures.

Methods: This study was conducted in Erbil from May 2013 till March 2015. Injured flexor tendons were repaired for 121 patients. The patients were followed up for six months. We used the Strickland scoring system to evaluate the results.

Results: Sharp tools represented the main cause of trauma $68.6 \%$, excellent results were found in $51.8 \%$ of cases with a sharp injury. The worst results were obtained in zone II with $44.7 \%$ fair to a poor result. Excellent results found in $43.4 \%$ with four strand core sutures while $24.4 \%$ in two strand core sutures $(P<0.01)$.

Conclusion: Four strand core sutures have the better result with a low rate of tendon rupture than two strand core sutures. Sharp injuries had better results than crush injuries. The zone with worst results was zone II.

Keywords: Flexor tendon; Core suture; Zone of injury; Mechanism of injury.

\section{Introduction}

Flexor tendons are essential to hand function, playing a vital role in all types of grip, including power grip and fine pinch grip. Flexor tendon injuries frequently occur through division in deep lacerations of the fingers, palm, or forearm. ${ }^{1}$ Flexor tendon injuries commonly occur in young, working people. ${ }^{2}$ The most common mechanism of

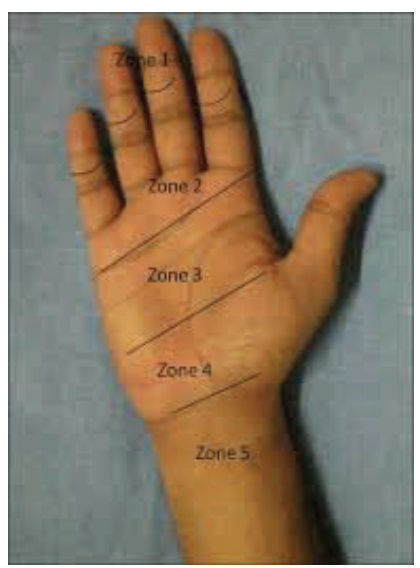

Figure 1: Flexor tendon zones (5 zones). finger flexor tendon disruptions reported in children is cut by glass. ${ }^{3}$ superior function obtained in the repair of sharply incised tendons as opposed to crushing injuries is a well-known fact. ${ }^{4}$ Flexor tendon injuries classified into five distinct zones as shown in Figure 1. Pulley system consists of five annular pulleys and three cruciate pulleys as shown in Figure $2 .^{5}$

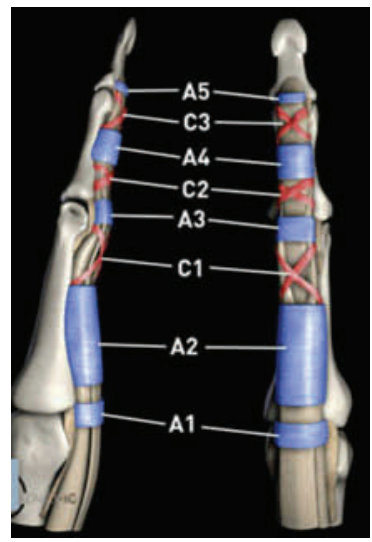

Figure 2: Flexor tendon sheath (5 annular and 3 cruciate pullies)

* Department of Surgery, College of Medicine, Hawler Medical University, Erbil, I raq. 
Since 1918, when Bunnell first described the concept of the "No Man's Land," zone II flexor tendon repair has remained challenging. Despite almost 100 years of research evaluating primary flexor tendon repair, hand surgeons continue to search for the optimal repair method. ${ }^{6}$ Since the 1960s the surgical technique, the basic science and the rehabilitation of flexor tendon repair have been the subject of numerous scientific studies both in vivo and ex vivo. ${ }^{7}$ Primary repair of the flexor tendon in the digital sheath area was established in the 1970s to the $1980 \mathrm{~s}^{8}$ Principles of surgical treatment of flexor tendon injuries include early primary repair with a strong core stitch combined with an epitendinous suture. ${ }^{9}$ The best results of tendon surgery often rely on the condition of the tissues, patient comorbidities and commitment injury, and the initial surgical technique. ${ }^{10}$ The Strickland described the characteristics of an ideal primary flexor tendon repair: easily placed in the tendon, secure knots, smooth junctions, minimal gapping, minimal interference with tendon vascularity, sufficient strength throughout healing to permit application of early motion stress. The ideal core suture material should have high tensile strength, be inextensible, cause no tissue reaction, and be easy to handle and knot. $^{11}$ Postoperative early active mobilization holds many advantages over passive mobilization protocols. $^{12}$ Increasing the number of suture strands crossing the repair site increases strength and resistance to gap formation. ${ }^{13}$ Active motion is started as soon as the patient has recovered from the anesthesia. Tendon repair takes about three months to achieve full strength. ${ }^{14}$ Careful selection of the postoperative splint and exercise regimen is as important as the repair technique. ${ }^{15}$ We conducted this study since flexor tendon injury is a common trauma in our city, yet little study has been done on this important topic. This study aimed to evaluate the outcomes of primary flexor tendon repair according to the number of core sutures, the mechanism of injury and the zone of injury.

\section{Methods}

\section{Design and Setting of the Study}

A prospective study of 121 cases (total 203 tendons) with flexor tendon injury that underwent flexor tendon repair between May 2013 and March 2015 with at least 6-month follow-up conducted at Rozhawa and Rozhhalat Emergency hospitals. The review aims to show the outcome of flexor tendon repair according to the mechanism of injury, the zone of injury and the number of core suture.

A questionnaire designed which included: demographic data, mechanism of injury, hand dominance, finger involved, associated injury, the zone of injury, tendon involved, partial or complete cut, TAM (total active motion), complications: early and late, patient and surgeon satisfaction. The patients were followed up for six months. In the first three months the follow up was done on weekly bases and in the last three months, it was done on monthly bases. During the follow-up range of movement, complications, and patient satisfaction were recorded.

\section{Exclusion criteria}

The exclusion criteria included fingers with concomitant fractures, associated with extensor tendon injury, associated with concomitant nerve injury and uncooperative patients.

\section{Procedure}

The repair was done by modified Kessler technique and sutured by 3.0 or 4.0 polypropylene with two or four strand core suture with continuous running suture with 6.0 polypropylene as shown in Figure 3. For zone II only profundus tendon was repaired, with preservation of the pulley system A2 and A4. For zones III,IV and $\mathrm{V}$ both flexor digitorum profundus and superficialis were repaired. Post-operative splinting for 3-4 weeks with Kleinert (69 cases) or static splint (52 cases) as 
https:/ / doi.org/ 10.15218/ zjms.2018.001

shown in Figure 4 and 5 . We used Strickland's criteria to evaluate functional recovery of flexor tendon repairs which were divided into four groups; excellent, good, fair and poor. Follow up and assessments of the range of finger flexion were done using goniometer as shown in Figure 6.

\section{Ethical consideration}

An informed written consent written in the mother tongue language of the participants using simple non-medical words, taken from those above 18 years old and patient's parents for those below this age.

Data management and statistical analysis

Data were analyzed using the statistical package of the social sciences (version 19) and the results were compared between the patients with different variables. A $P$ value of $\leq 0.05$ was considered statistically significant.

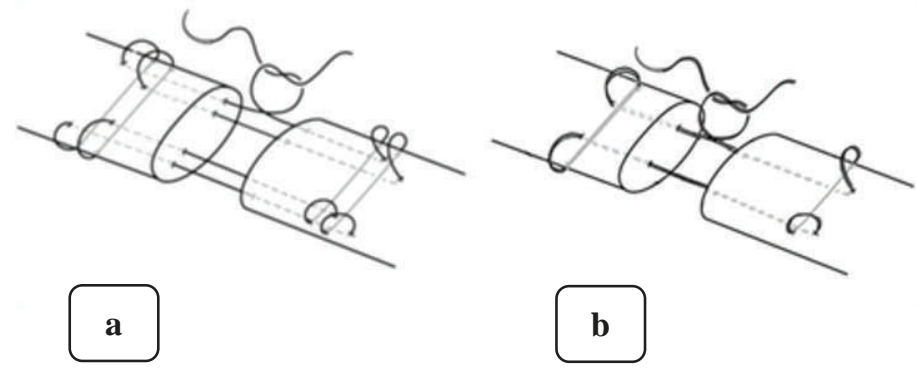

Figure 3: a- Four strand core suture. b- Two strand core suture.

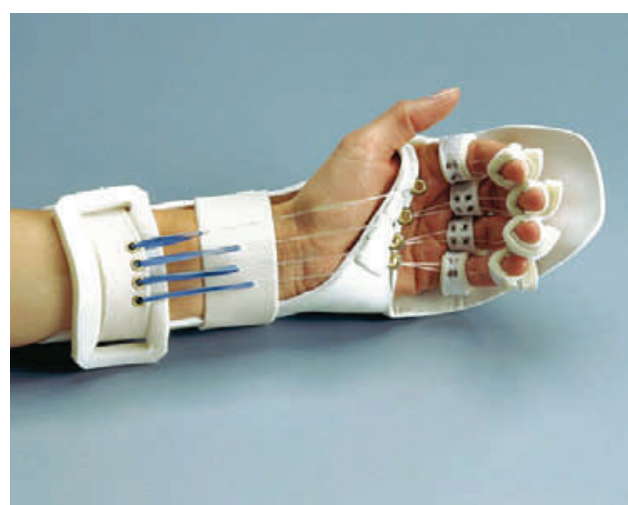

Figure 4: Kleinert splint.

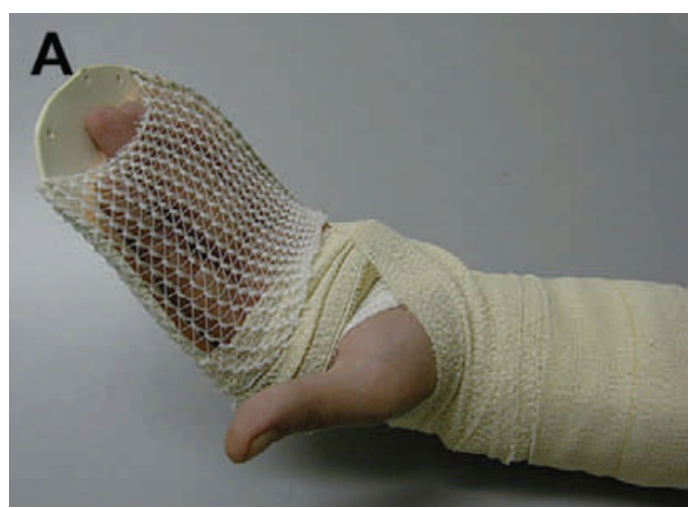

Figure 5: Static splint.

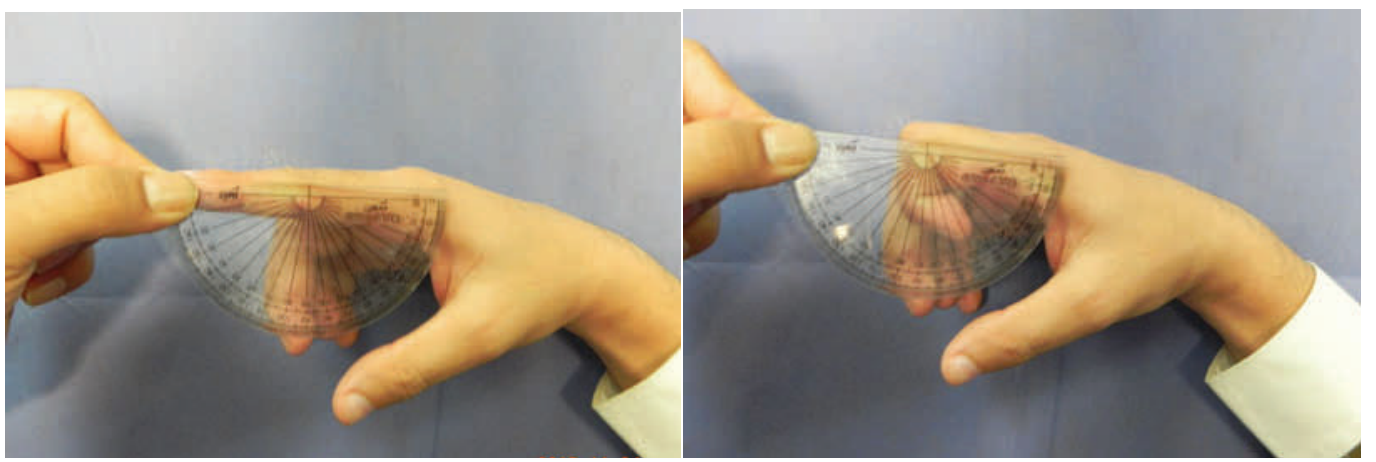

Figure 6: Assessment of degree of finger flexion and extension with goniometer. 


\section{Results}

In this study, the total cases were 121 patients with 203 flexor tendon repair. The mean age was $27 \pm$ SD13.16 years. The gender distribution was $85(70.2 \%)$ male and $36(29.8 \%)$ females. The commonest occupation was unskilled manual workers (86 cases, $71.1 \%$ ). Sharp tools represented the main cause of trauma (83 cases, $68.6 \%$ ) as shown in Table 1. Sharp injuries had a better result than crush injuries with $P=0.003$ which is highly significant statistically as shown in Table 2 . The best results were obtained in zone 4 and 3 , with $\mathrm{P}=0.004$ as shown in Table 3.

Table 1: Mechanism of injury.

\begin{tabular}{lcc}
\hline Mechanism of injury & Frequency & Percent \\
\hline Sharp & 83 & 68.6 \\
Crush & 38 & 31.4 \\
Total & 121 & 100 \\
\hline
\end{tabular}

Table 2: Results according to the mechanism of injury.

\begin{tabular}{llccccc}
\hline \multirow{2}{*}{ Injury mechanism } & & Result & & Total \\
& & excellent & good & fair & poor & \\
\hline Sharp & No. & 43 & 36 & 3 & 1 & 83 \\
& $\%$ & 51.8 & 43.4 & 3.6 & 1.2 & 100.0 \\
\multirow{3}{*}{ Crush } & No. & 1 & 10 & 18 & 9 & 38 \\
& $\%$ & 2.6 & 26.3 & 47.4 & 23.7 & 100.0 \\
\multirow{2}{*}{ Total } & No. & 44 & 46 & 21 & 10 & 121 \\
& $\%$ & 36.4 & 38.0 & 17.4 & 8.3 & 100.0 \\
\hline
\end{tabular}

Table 3: Results according to the zone of injury.

\begin{tabular}{|c|c|c|c|c|c|c|}
\hline \multirow{2}{*}{\multicolumn{2}{|c|}{ Zone of injury }} & \multicolumn{4}{|c|}{ Result } & \multirow{3}{*}{$\begin{array}{r}\text { Total } \\
20\end{array}$} \\
\hline & & \multirow{2}{*}{$\frac{\text { excellent }}{9}$} & \multirow{2}{*}{$\frac{\text { good }}{8}$} & \multirow{2}{*}{$\begin{array}{c}\text { fair } \\
2\end{array}$} & \multirow{2}{*}{$\frac{\text { poor }}{1}$} & \\
\hline zone 1 & No. & & & & & \\
\hline & $\%$ & 45.0 & 40.0 & 10.0 & 5.0 & 100.0 \\
\hline \multirow[t]{2}{*}{ zone 2} & No. & 3 & 28 & 16 & 9 & 56 \\
\hline & $\%$ & 5.4 & 50.0 & 28.6 & 16.1 & 100.0 \\
\hline \multirow[t]{2}{*}{ zone 3} & No. & 15 & 3 & 1 & 0 & 19 \\
\hline & $\%$ & 78.9 & 15.8 & 5.3 & .0 & 100.0 \\
\hline \multirow[t]{2}{*}{ zone 4} & No. & 8 & 1 & 1 & 0 & 10 \\
\hline & $\%$ & 80.0 & 10.0 & 10.0 & .0 & 100.0 \\
\hline \multirow[t]{2}{*}{ zone 5} & No. & 9 & 6 & 1 & 0 & 16 \\
\hline & $\%$ & 56.3 & 37.5 & 6.3 & .0 & 100.0 \\
\hline \multirow[t]{2}{*}{ Total } & No. & 44 & 44 & 46 & 21 & 121 \\
\hline & $\%$ & 36.4 & 36.4 & 38.0 & 17.4 & 100.0 \\
\hline
\end{tabular}


https:/ / doi.org/ 10.15218/ zjms.2018.001

According to the number of core sutures, a better result was found with four core sutures as shown in Table 4. The commonest complication was adhesion (18 cases, 14.9\%) as shown in Table 5.

\section{Discussion}

The aim of flexor tendon repair is the near-complete restoration of range of active digital flexion while avoiding rupture of repair and formation of serious adhesion or joint contracture. Reporting gender distribution of cases varied in the literature; as some studies neglected reporting it, others considered reporting it. In this study, there were 85 male patients representing $70.2 \%$ of cases which is consistent with a study done by Christopher et al. $^{2}$ and Matthias et al. ${ }^{8}$ study. The high percentage of male patients' affection is logically due to the hard nature of activities of male patients. Excellent to good results obtained in $74.4 \%$, and this is consistent with a study done by Rudge and James ${ }^{11}$ who did primary flexor tendon repair of 209 flexor tendons and $72.4 \%$ had excellent to good results and Peter et al. who showed that two thirds of primary flexor tendon repairs had good and excellent results. ${ }^{5}$ The relationship between the zone of injury and outcome is an important concept. It is well known that the functional outcome of zone II flexor tendon injuries is poorer and is associated with a complication rate greater than that of injuries in other zones. The most common complication after surgical repair of tendon injuries is adhesion formation. This is of particular concern in the setting of zone II injuries because of the unique anatomy of the flexor tendon sheath in this region. As the FDS and FDP tendons are encased in a narrow fibro -osseous tunnel, even slight bulkiness of the flexor tendon or minimal adhesion formation may result in a significant increase in friction. Thus, minimal anatomic changes within the fibro-osseous tunnel may result in marked limitation of tendon

Table 4: Result according to core suture technique.

\begin{tabular}{|c|c|c|c|c|c|c|}
\hline \multirow{2}{*}{\multicolumn{2}{|c|}{ Suture technique }} & \multicolumn{4}{|c|}{ Result } & \multirow{3}{*}{$\begin{array}{r}\text { Tota } \\
45\end{array}$} \\
\hline & & \multirow{2}{*}{$\frac{\text { excellent }}{11}$} & \multirow{2}{*}{$\frac{\text { good }}{14}$} & \multirow{2}{*}{$\begin{array}{r}\text { fair } \\
14\end{array}$} & \multirow{2}{*}{$\frac{\text { poor }}{6}$} & \\
\hline Two strand & No. & & & & & \\
\hline & $\%$ & 24.4 & 31.1 & 31.1 & 13.3 & 100.0 \\
\hline \multirow[t]{2}{*}{ Four strand } & No. & 33 & 32 & 7 & 4 & 76 \\
\hline & $\%$ & 43.4 & 42.1 & 9.2 & 5.3 & 100.0 \\
\hline \multirow[t]{2}{*}{ Total } & No. & 44 & 46 & 21 & 10 & 121 \\
\hline & $\%$ & 36.4 & 38.0 & 17.4 & 8.3 & 100.0 \\
\hline
\end{tabular}

Table 5: Frequency and percentage of Complications.

\begin{tabular}{lcc}
\hline Complication & Frequency & Percent \\
\hline No Complications & 71 & 58.7 \\
Adhesion & 18 & 14.9 \\
Quadriga & 11 & 9.1 \\
Rupture & 9 & 7.4 \\
Joint stiffness & 5 & 4.1 \\
Infection & 4 & 3.3 \\
Lumbrical plus & 3 & 2.5 \\
Total & 121 & 100 \\
\hline \hline
\end{tabular}


https:/ / doi.org/ 10.15218/ zjms.2018.001

excursion and compromised function. Factors that have been demonstrated to affect the formation of excursion-restricting adhesions postoperatively are trauma to the tendon and sheath from the initial injury and surgical repair, tendon ischemia, immobilization, and gapping at the tendon repair site. In this study, the zone with worst results was zone II with $44.7 \%$ fair to poor result, which is compliant with other studies by Trevor et al. ${ }^{10}$ and David et al. ${ }^{14}$ According to the mechanism of injury, sharp injuries had better results than crush injuries. Excellent results were found in $51.8 \%$ of cases with sharp injury while in crush injury only $2.6 \%$ of cases, with $P<0.01$ and this result is consistent with a study done by Trevor et al. ${ }^{10}$ and Brian et al. $^{7}$ According to the number of strand core sutures, the result was superior with four core strand sutures as excellent to good result were found in $85.5 \%$ with no rapture rate with 4 strand core sutures (Figure 7 and 8 ),
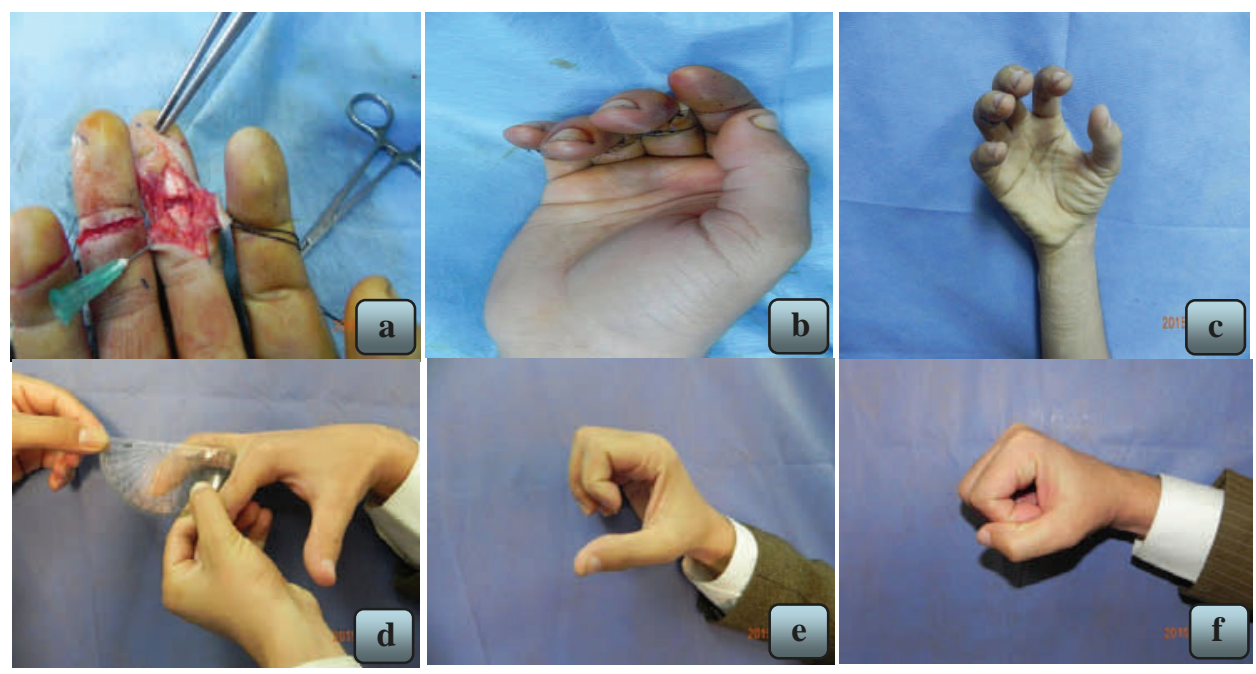

Figure 7: Zone 1 injury a- flexor digitorum profundus cut of right middle and ring finger before repair, b- after repair( four strand core suture+ epitendinous suture), c- one month postoperative, d- assessment of the range of movement with goniometer, e \& f- shows excellent result.
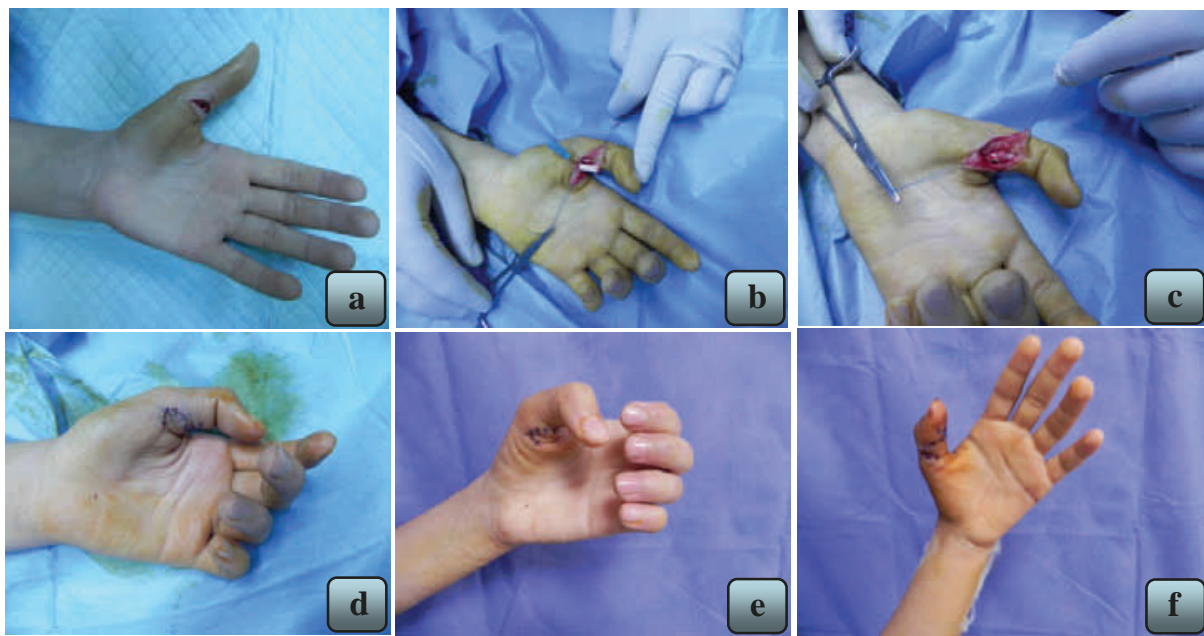

Figure 8: a- zone two injury of left thumb, b- the proximal stump of the cut flexor policis tendon has been retrieved and fixed with a needle, c- repair done(four strand core suture+ epitendinous suture) with slight overcorrection, $d$, e and $f$ - after splint removal shows good result. 
while excellent to good result found in only $55.5 \%$ with $20 \%$ rapture rate with two strand core sutures, i.e. more strand core sutures had better outcome and less complications. This result is consistent with Peter et al., ${ }^{5}$ Arash et al., ${ }^{6}$ Brian et al., Matthias et al., ${ }^{8}$ and Jin et al. ${ }^{16}$ In our study post-operative early active movement had a better result with a low rate of complications like tendon adhesion with the use of Kleinert splint which is consistent with the study of Brian et al., ${ }^{7}$ Rudge $^{11}$ and Maurizio et al. ${ }^{12}$

\section{Conclusion}

Four strand core sutures have a better result with a low rate of tendon rupture than two strand core sutures. Sharp injuries had better results than crush injuries. The zone with worst results was zone II. Kleinert splints had better results than static splints. Four strand core suture is recommended for better results. We recommend intraoperative testing of the repair for possible gapping. We recommend for the $\mathrm{MOH}$ to supply the emergency hospitals with Kleinert splints so that the poor people can get benefit from the advantages of that splint for a better result. We advise for further study on this important topic on a larger sample size and longer follow up.

\section{Competing interests}

The authors declare that they have no competing interests.

\section{References}

1. Frank V, Chris J, Nick L, Mark G, William W. Stress relaxation and creep viscoelastic properties of common suture materials used for flexor tendon repair. JHS 2008; 33:241-6.

2. Christopher D, Aaron D, Huong D, Alexia S. Robert M, Stephen L. The epidemiology of reoperation after flexor tendon repair. JHS 2012; 37:919-24.

3. Yrjana N, Nina L, Sauli P, Sanna R, Nadja R. Flexor tendon injuries in pediatric patients. JHS 2007; 32:1549-57.

4. Trevor S, Rebecca S, Kenneth M. Clinical outcomes of zone II Flexor tendon repair depending on mechanism of injury. JHS 2012; 37:2532-40.
5. Marissa R, Warren C. Flexor Tendon Rehabilitation. JHS 2012; 37A:2386-8.

6. Mordechai V, Ram P, Rachel G, Vipul P, Phillip N, Steve L. Biomechanical study of cross-locked cruciate versus Strickland flexor tendon repair. JHS 2008; 33:1826-33.

7. Atwal N, Sheriff D, Cawey A. Flexor tendon injuries. OAT 2010; 24: 217-22.

8. Jin T, Peter A, Martin B, Robert S, Chunfeng Z, Michael $S$, et al. Current practice of primary flexor tendon repair a global view. HC 2013; 21: 1-11.

9. Arash M, Emily G, James C. Complications after flexor tendon injuries. HC 2009; 26:179-89.

10. Brian M, Bradon J, Elvin G, Michael W. Flexor tendon reconstruction. CPS 2011;38:607-19.

11. Rudge J, James M. Flexor tendon injuries in the hand a UK survey of repair techniques and suture materials are we following the evidence. ISRN PS 2014; 1:1-4.

12. Maurizio C, Monia B, Marco F, Galeazzo C, Silvio T, Francesco P. The four-strand staggered suture for flexor tendon repair in Vitro biomechanical study. JHS 2010; 32:948-55.

13. Mike H, Gregory $N$, Stavros $T$, Matthew S, Rosalina D, Richard G. Technical and biological modifications for enhanced flexor tendon repair. JHS 2010; 35:1031-7.

14. David E, Thomas G. Primary flexor tendon surgery the search for a perfect result. HC 2013; 29:191-206.

15. Julianne $H$, Fiona $P$. Rehabilitation of flexor and extensor tendon injuries in the hand. IJCI 2013; 44:397-402.

16. Jin T. Outcomes and evaluation of flexor tendon repair. HC 2013; 29:251-9. 